\title{
The Central Stars Parameters and the Distances to Galactic Planetary Nebulae
}

\author{
O.I. Sharova
}

Radio physical Research Institute, Nizhny Novgorod, 603950, Russia

In our paper (Sharova, 1995) a method of determining the distances to galactic planetary nebulae has been based on the recently established (Sharova, 1992) regression relationship between the central stars radius and the temperature. Now our scale is compared to 18 other scales, in four of which the central stars parameters also are used, instead of nebulae ones. The scale distance estimations $D_{S}$ are compared with independently known individual distance $D$. The values $\log \left(D_{S} / D\right)$ and their dependence on radius of the nebulae are analysed. Each scale is described by two parameters: a geometrical average value $\left\langle\log D_{S} / D\right\rangle$ and the relation $\rho / \rho_{c}$, where $\rho$ is the correlation coefficient for $\log D_{S} / D$ $\log R_{N}$, and $\rho_{c}$ is its critical value. If $\rho / \rho_{c}>1$, the correlation is significant with the confidence probability $95 \%$. For example we obtained the following results: $\rho / \rho_{c}=0.153,0.494,2.126,2.228 ;\left\langle D_{S} / D\right\rangle=0.999,2.145,2.126,1.414$ for our scale and for widely known MKH, CKL, VdSZ scales respectively. By both criteria our scale achieves better agreements, on average, with individual estimations of distances than others.

We have derived the hydrogen density $\left(N_{\mathrm{H}}\right)$, the ionized mass $M_{i}$ and the kinematical ages $t$ for 132 planetary nebulae based on our distances. We obtained the following regression relationships:

$$
\log N_{\mathrm{H}}\left(\mathrm{cm}^{-3}\right)=8.51-1.49 \log t(\mathrm{yr}) ; \quad \log M_{i}\left(\mathrm{M}_{\odot}\right)=-6.23+1.42 \log t(\mathrm{yr})
$$

The empirical relationships agree with the theoretical ones in the energyconserving case of ISW model, if mass loss rate from central star is constant and equals to $1-5 \times 10^{-8} \mathrm{M}_{\odot} \mathrm{yr}^{-1}$. We have also calculated the evolution of radio continuum spectra using parameters of ISW model best fitted empirical evolution relationships and found that the radio flux density is increasing while $t \lesssim 1000$ years in all central star models except for $0.836 M_{\odot}$. The rate of the radio flux change in model with $0.646 M_{\odot}$ may achieve $8 \% / \mathrm{yr}$.

\section{References}

Sharova, O.I., 1995, Radiophysics and Quantum Electronics, 38, 344.

Sharova, O.I., 1992, Soviet Ast., 36, 18. 